\title{
Hereditary Hemochromatosis (Clinical case)
}

\author{
Hemocromatose hereditária (Caso clínico) \\ Bruno Sousa ${ }^{1,2,3}$, Nelson Tavares ${ }^{1,2^{*}}$
}

\begin{abstract}
${ }^{1}$ School of Sciences and Health Technologies, Universidade Lusófona de Humanidades e Tecnologias, Lisboa, Portugal
${ }^{2}$ CBIOS - Universidade Lusófona's Research Center for Biosciences and Health Technologies, Lisboa, Portugal

${ }^{3}$ Health Service of Autonomous Region of Madeira, Madeira, Portugal

* corresponding author (current affiliation) / autor para correspondência (afiliação actual): nelsontavares@natiris.pt

Received / Recebido: 10/07/2021 Accepted / Aceite: 24/10/2021

Electronic Edition: www.alies.pt
\end{abstract}

A sixty-eight-year-old retired man was sent for Nutrition Consultation by his family doctor due to hereditary hemochromatosis (homozygosity H63D) and other pathologies that require nutritional therapy. He was also sent to a Transfusion Medicine and Rheumatology Consultation.

Keywords: hereditary hemochromatosis, excess iron absorption, organ and tissue damage

\section{Anthropometric assessment}

Weight: $109.8 \mathrm{~kg}$

Height: $170 \mathrm{~cm}$

BMI: $38 \mathrm{~kg} / \mathrm{m}^{2}$

Waist circumference: $129 \mathrm{~cm}$

\section{Body composition assessment (TANITA TBF 300 ${ }^{\circledR}$ )}

Body fat: $45.8 \%$

Fat mass: $50.3 \mathrm{~kg}$

Fat-free mass: $59.5 \mathrm{~kg}$

Total body water: $43.6 \mathrm{~kg}$

\section{Analytical Parameters}

\section{Hematology}

Leukocytes: $9.010^{\wedge} 3 / \mu \mathrm{L}(4.5-110)$

Erythrocytes: $4.5910^{\wedge} 6 / \mu \mathrm{L}(4.50-6.50)$

Hemoglobin: 14.9 g/dL $(13.0-18.0)$

Hematocrit: $44.0 \%$ (40.0 - 54.0)

Glycated hemoglobin (A1C): 5.8\% (4.0 - 6.0)
Um homem de 68 anos, reformado, foi enviado à Consulta de Nutrição pela médica de família por hemocromatose hereditária (homozigotia H63D) e com outras patologias que necessitam de terapêutica nutricional. É enviado também para a Consulta de Medicina Transfusional e de Reumatologia.

Palavras-chave: hemocromatose hereditária, absorção excessiva de ferro, danos de órgãos e tecidos

\section{Avaliação antropométrica}

Peso: $109,8 \mathrm{~kg}$

Estatura: $170 \mathrm{~cm}$

IMC: $38 \mathrm{~kg} / \mathrm{m}^{2}$

Perímetro da cintura: $129 \mathrm{~cm}$

Avaliação da composição corporal (TANITA TBF 300 ${ }^{\circledR}$ )

Gordura corporal: 45,8\%

Massa gorda: $50,3 \mathrm{~kg}$

Massa isenta de gordura: $59,5 \mathrm{~kg}$

Água corporal total: $43,6 \mathrm{~kg}$

\section{Parâmetros analíticos}

\section{Hematologia}

Leucócitos: 9,0 10^3/ $\mu \mathrm{L}(4,5$ - 110)

Eritrócitos: 4,59 10^6/ $\mu \mathrm{L}(4,50-6,50)$

Hemoglobina: $14,9 \mathrm{~g} / \mathrm{dL}(13,0-18,0)$

Hematócrito: 44,0\% (40,0 - 54,0)

Hemoglobina glicada (A1C): 5,8\% (4,0 - 6,0) 
Prothrombin time: $27.9 \mathrm{sec}(9.4-12.5)$

INR: 2.34 (0.9-1.2)

\section{Biochemistry}

Glucose: $97 \mathrm{mg} / \mathrm{dL}(82.0$ - 115.0)

Urea: $43 \mathrm{mg} / \mathrm{dL}(8.0-50.0)$

Creatinine: $1.07 \mathrm{mg} / \mathrm{dL}(0.70-1.20)$

Uric acid $6.4 \mathrm{mg} / \mathrm{dL}(4.8-8.0)$

Total cholesterol: $180 \mathrm{mg} / \mathrm{dL}(<200.0)$

HDL cholesterol: $32.0 \mathrm{mg} / \mathrm{dL}(>40.0)$

LDL cholesterol: $120.8 \mathrm{mg} / \mathrm{dL}(<115.0)$

Triglycerides: $136.0 \mathrm{mg} / \mathrm{dL}(<150.0)$

Alanine Aminotransferase: 55.7 U/L (17.0 - 63.0)

Aspartate Aminotransferase: $40.6 \mathrm{U} / \mathrm{L}(10.0-50.0)$

Gamaglutamyltransferase: $45.6 \mathrm{U} / \mathrm{L}(7.0-50.0)$

Iron: $130.0 \mu \mathrm{g} / \mathrm{dL}(45.0$ - 182.0)

C-reative protein: $2.98 \mathrm{mg} / \mathrm{L}(<6.10)$

\section{Hormonology}

Thyroid function:

Free T4: $1.3 \mathrm{ng} / \mathrm{dL}(0.6$ - 1.7)

TSH: $1.39 \mu \mathrm{UI} / \mathrm{mL}(0.30$ - 4.70)

\section{Anemias}

Ferritin: $286.0 \mathrm{ng} / \mathrm{mL}(30.0$ - 400.0)

Folic acid: $5.45 \mathrm{ng} / \mathrm{mL}(>3.89)$

Vitamin B12: 563 pg/ml (197 - 771)

\section{Clinical evaluation}

\section{Personal background}

- Acute myocardial infarction approximately three years ago

- Mixed dyslipidemia

- Severe hepatic steatosis

- Atrial Fibrillation

- HTA

- Class II obesity

- Obstructive Sleep Apnea Syndrome

\section{Medication}

Omeprazol $20 \mathrm{mg}$; Paracetamol $1 \mathrm{~g}$; Atorvastatina $20 \mathrm{mg}$; Enalapril/hidroclorotiazida $20 \mathrm{mg} / 12,5 \mathrm{mg}$; Sintrom $4 \mathrm{mg}$; Bisoprolol 2,5 mg; Fenofibrato $145 \mathrm{mg}$; Metformina $850 \mathrm{mg}$.
Tempo de protrombina: $27,9 \mathrm{seg}(9,4-12,5)$

INR: 2,34 $(0,9-1,2)$

\section{Bioquímica}

Glicose: $97 \mathrm{mg} / \mathrm{dL}(82,0$ - 115,0)

Ureia: $43 \mathrm{mg} / \mathrm{dL}(8,0$ - 50,0)

Creatinina: $1, .07 \mathrm{mg} / \mathrm{dL}(0,70-1,20)$

Ácido úrico: $6,4 \mathrm{mg} / \mathrm{dL}(4,8-8,0)$

Colesterol total: $180 \mathrm{mg} / \mathrm{dL}(<200,0)$

Colesterol HDL: $32,0 \mathrm{mg} / \mathrm{dL}(>40,0)$

Colesterol LDL: $120,8 \mathrm{mg} / \mathrm{dL}(<115,0)$

Triglicéridos: $136,0 \mathrm{mg} / \mathrm{dL}(<150,0)$

Alanina Aminotransferase: 55,7 U/L (17,0 - 63,0)

Aspartato Aminotransferase: 40,6 U/L (10,0 - 50,0)

Gamaglutamiltransferase: 45,6 U/L (7,0 - 50,0)

Ferro: $130.0 \mu \mathrm{g} / \mathrm{dL}(45,0$ - 182,0)

Proteína C-reativa: $2,9 \mathrm{mg} / \mathrm{L}(<6,10)$

Hormonologia

Função tiroideia:

T4 livre: $1,3 \mathrm{ng} / \mathrm{dL}(0,6-1,7)$

TSH: $1,39 \mu \mathrm{UI} / \mathrm{mL}(0,30-4,70)$

Anemias

Ferritina: $286,0 \mathrm{ng} / \mathrm{mL}(30,0$ - 400,.0)

Ácido fólico: $5,45 \mathrm{ng} / \mathrm{mL}(>3,89)$

Vitamina B12: 563 pg/mL (197 - 771)

\section{Avaliação clínica}

Antecedentes pessoais:

- Enfarte agudo do miocárdio há cerca de 3 anos

- Dislipidemia mista

- Esteatose hepática severa

- Fibrilação Auricular

- HTA

- Obesidade classe II

- Síndrome de Apneia Obstrutiva do Sono

Medicação:

Omeprazol 20 mg; Paracetamol $1 \mathrm{~g}$; Atorvastatina 20 mg; Enala-pril/hidroclorotiazida $20 \mathrm{mg} / 12,5 \mathrm{mg}$; Sintrom $4 \mathrm{mg}$; Bisoprolol 2,5 mg; Fenofibrato $145 \mathrm{mg}$; Metformina $850 \mathrm{mg}$. 
Complementary diagnostic tests

- NMR (2013): Left sacroiliitis

- Joint ultrasound (October 2017): no signs of active synovitis

- Electrocardiogram (July/2018): no significant changes

- Echocardiogram (July/2018): examination made difficult by poor acoustic window, mild mitralaortic degenerative changes + slight left ventricular hypertrophy with preserved systolic function.

Intestinal transit: regular

\section{Eating habits}

Wake up at 7 am

Breakfast: 7:30 am

1 cup of semi-skimmed milk $(240 \mathrm{ml})$ with instant blended coffee +1 dessert spoon of sugar

2 buns +1 slice of cheese +1 slice of ham

Lunch: 1:00 pm

Plate: Half a plate of white rice or 3 potatoes + meat or fish (120 g) + cooked vegetables (about $100 \mathrm{~g}$ )

Drink beer or water

Afternoon snack: 4:30 pm

1 cup of semi-skimmed milk $(240 \mathrm{ml})$ with instant blended coffee +1 dessert spoon of sugar

2 buns +1 slice of cheese +1 slice of ham

Dinner: 8:00 pm

Identical to lunch

Bedtime snack: 10:30 pm

1 glass of semi-skimmed milk $(200 \mathrm{ml})$

3 Maria cookies

Go to bed at 11:00 pm

Water consumption: about 1 liter per day

Alcohol habits: about 6 beers a day

Smoking habits: Stopped smoking approximately five years ago
Exames complementares de diagnóstico

- RMN (2013): Sacroileíte esquerda

- Ecografia articular (outubro/2017): sem sinais de sinovite ativa

- Electrocardiograma (julho/2018): sem alterações significativas

- Ecocardiograma (julho/2018): exame dificultado por má janela acústica, ligeiras alterações degenerativas mitro-aórticas + ligeira hipertrofia do ventrículo es-querdo com função sistólica preservada.

Trânsito intestinal: regular

\section{Hábitos alimentares}

Acorda às $7 \mathrm{~h}$

Pequeno-almoço: $7 \mathrm{~h} 30$

1 chávena de leite meio gordo $(240 \mathrm{ml})$ com café de mistura solúvel +1 colher de sobremesa de açúcar 2 pães +1 fatia de queijo +1 fatia de fiambre

Almoço: $13 \mathrm{~h}$

Prato: Meio prato de arroz branco ou 3 batatas + carne ou peixe $(120 \mathrm{~g})+$ verdura cozidas (cerca de $100 \mathrm{~g}$ )

Bebe cerveja ou água

Lanche da tarde: $16 \mathrm{~h} 30$

1 chávena de leite meio gordo $(240 \mathrm{ml})$ com café de mistura solúvel +1 colher de sobremesa de açúcar

2 pães +1 fatia de queijo +1 fatia de fiambre

Jantar: $20 \mathrm{~h}$

Idêntico ao almoço

Ceia: $22 \mathrm{~h} 30$

1 copo de leite meio gordo $(200 \mathrm{ml})$

3 bolachas Maria

Deita-se às $23 \mathrm{~h}$

Consumo de água: cerca de 1 litro por dia

Hábitos etílicos: cerca de 6 cervejas diárias

Hábitos tabágicos: deixou de fumar há cerca de 5 anos. 


\section{Environment, behavior and social}

He is married and lives with his wife.

He spends most of his time at home, where he takes his meals, and is not in the habit of socializing with people other than his household.

He likes to eat bread and mentions that he is careful in the preparation of food, namely, to avoid fried foods.

He has a sedentary activity. He reports difficulty in performing certain activities of daily living, namely putting on shoes.

\section{Questions}

1. What is hereditary hemochromatosis?

2. How can hereditary hemochromatosis be identified?

3. What are the symptoms of hemochromatosis?

4. How can complications from hereditary hemochromatosis be prevented?

5. What dietary changes will help prevent complications?

\section{Authors Contributions Statement}

The contribution to the preparation of this Case Study was identical for both authors.

\section{Acknowledgements}

The authors wish to express their thanks to the patient who allowed the elaboration of the case study.

\section{Conflict of Interests}

The authors declare there are no financial and personal relationships that could present a potential conflict of interests.

\section{Ambiente, comportamento e social}

É casado e vive com a esposa.

Passa a maior parte do tempo em casa, onde realiza as suas refeições, e não tem por hábito conviver com outras pessoas para além do seu agregado familiar.

Gosta de comer pão e refere que tem cuidados na confeção dos alimentos nome-adamente a evitar os fritos.

Apresenta uma atividade sedentária. Refere dificuldade em executar determinadas atividades da vida diária, nomeadamente calçar os sapatos.

\section{Questões}

1. O que é hemocromatose hereditária?

2. Como a hemocromatose hereditária pode ser identificada?

3. Quais são os sintomas da hemocromatose?

4. Como prevenir as complicações da hemocromatose hereditária?

5. Como as que mudanças dietéticas ajudarão a prevenir complicações?

\section{Declaração sobre as contribuições do autor}

A contribuição na preparação deste Caso de Estudo foi idêntica para os dois autores.

\section{Agradecimentos}

Os autores desejam expressar os seus agradecimentos ao paciente que permitiu a elaboração desde e caso de estudo.

\section{Conflito de Interesses}

Os autores declaram que não há relações financeiras e pessoais que possam representar um potencial conflito de interesses. 


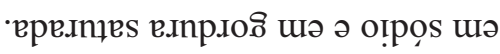

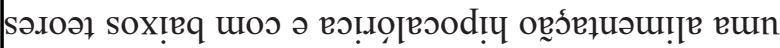

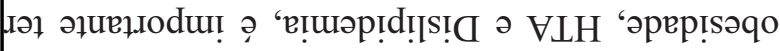

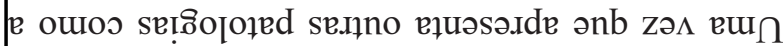

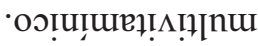

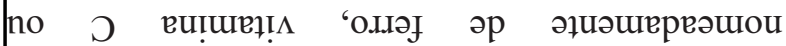

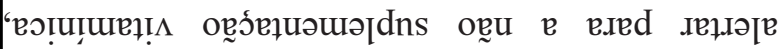

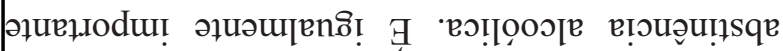

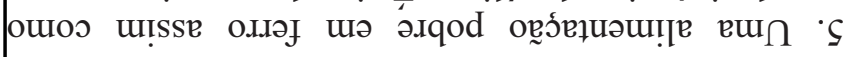

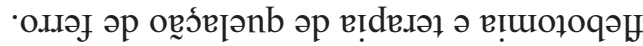

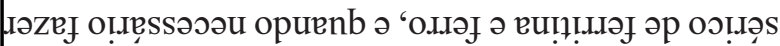

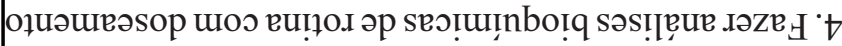

‘

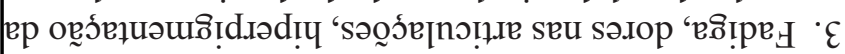

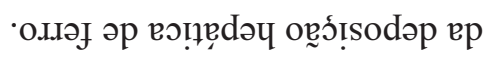

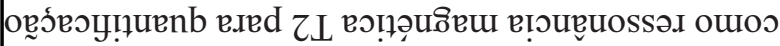

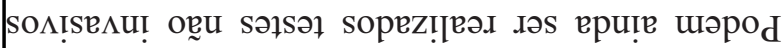

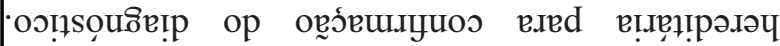

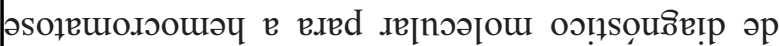

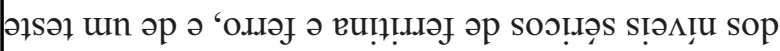

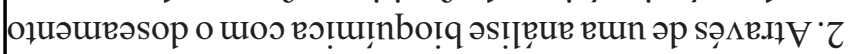

'oustpeuoôod!̣ ә

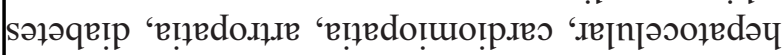

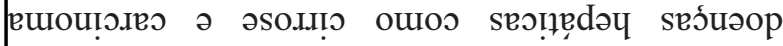

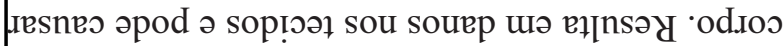

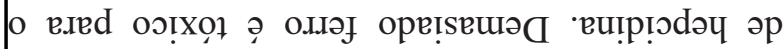

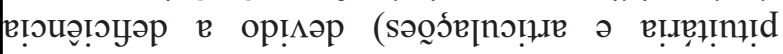

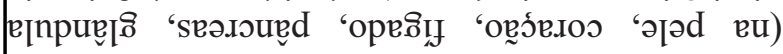

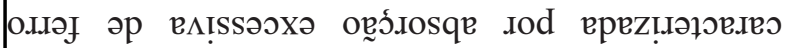

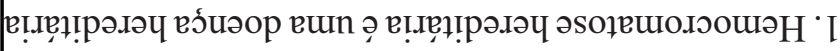

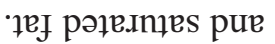

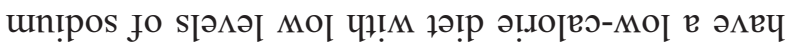

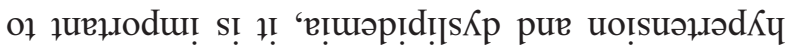
'Kı!

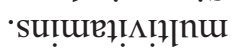

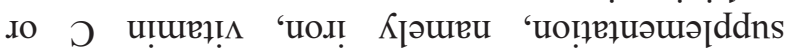

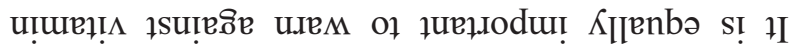

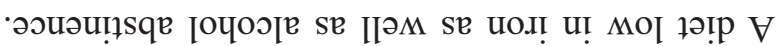

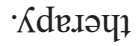

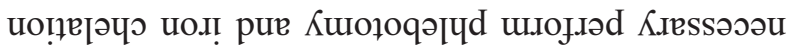

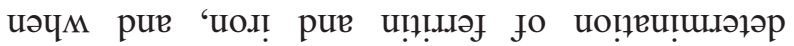

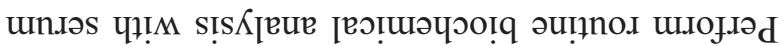

•əı!s

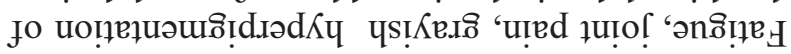

'uo!̣!̣sodəp uo.ı!

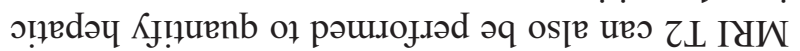

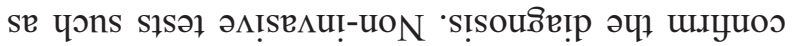

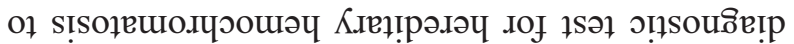

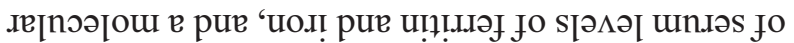

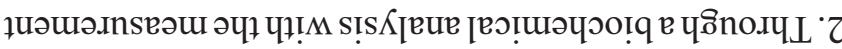

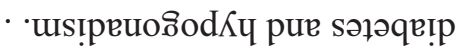

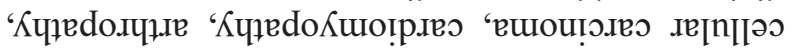

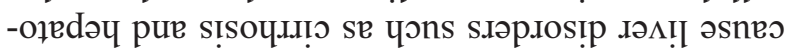

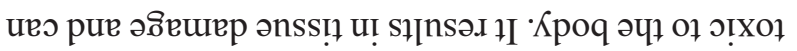

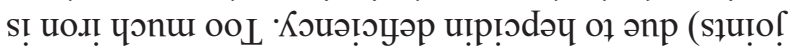

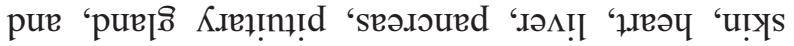

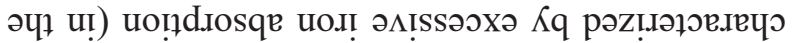

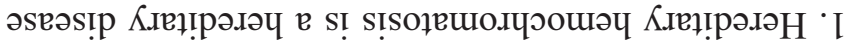




\section{References / Referências}

1. Kowdley, K. V., Brown, K. E., Ahn, J., \& Sundaram, V. (2019). ACG Clinical Guideline: Hereditary Hemochromatosis. The American journal of gastroenterology, 114(8), 1202-1218. https://doi.org/10.14309/ajg.0000000000000315

2. Porter, J. L., \& Rawla, P. (2021). Hemochromatosis. In StatPearls. StatPearls Publishing.PMID: 28613612.

3. Raymond, J.L., Morrow, K. (2020). Krause and Mahan's Food \& the Nutrition Care Process (15th ed). Saunders. 